\title{
A CONVERGENCE RESULT FOR THE PERIODIC UNFOLDING METHOD RELATED TO FAST DIFFUSION ON MANIFOLDS
}

\author{
Isabell Graf ${ }^{\mathrm{a}}$, Malte A. Peter ${ }^{\mathrm{b}, \mathrm{c}}$ \\ ${ }^{a}$ Department of Mathematics, Simon Fraser University, Burnaby BC, Canada \\ ${ }^{b}$ Institute of Mathematics, University of Augsburg, Augsburg, Germany \\ ${ }^{c}$ Augsburg Centre for Innovative Technologies, Augsburg, Germany
}

\begin{abstract}
Based on the periodic unfolding method in periodic homogenization, we deduce a convergence result for gradients of functions defined on connected, smooth and periodic manifolds. Under the assumption of certain a-priori estimates of the gradient, which are typical for fast diffusion, the sum of a term involving a gradient with respect to the slow variable and one with respect to the fast variable is obtained in the homogenization limit. In addition, we show in a brief example how to apply this result and find for a reaction-diffusion equation defined on a periodic manifold that the homogenized equation contains a term describing macroscopic diffusion.
\end{abstract}

\section{Résumé}

A l'aide de la méthode d'éclatement périodique, nous démontrons un résultat de convergence des gradients de fonctions définies sur des variétés connexes, différentiables et périodiques. Sous certaines conditions d'estimation du gradient, typiques de la diffusion rapide, nous obtenons à la limite d'homogénéisation la somme d'un gradient de la variable globale et d'un gradient de la variable locale. Un exemple illustre l'utilisation de ce résultat: pour une équation de réaction et diffusion définie sur une variété périodique, nous démontrons que l'équation homogénéisée contient un terme décrivant une diffusion globale.

Keywords: periodic homogenization, periodic unfolding method, fast diffusion, weak compactness result, smooth manifolds

2010 MSC: 35B27, 35K51

1. Setting. The periodic unfolding method is a technique to homogenize partial differential equations. The main idea is the introduction of an operator $\mathcal{T}_{\varepsilon}$, which maps a function $\varphi_{\varepsilon}$ defined on a finely structured periodic domain $\Omega_{\varepsilon} \subset \mathbb{R}^{n}$ to a function $\mathcal{T}_{\varepsilon}\left(\varphi_{\varepsilon}\right)$ defined on $\Omega \times Y$, where $Y=[0,1]^{n}$ is the periodicity cell. With $\Omega \subset \mathbb{R}^{n}$ being homogeneous, the domain of the function $\mathcal{T}_{\varepsilon}\left(\varphi_{\varepsilon}\right)$ is independent of $\varepsilon$ and hence, we are able to use well-known convergence results from functional analysis.

The periodic unfolding method was developed in $[3,4,5,6]$ based on ideas of [2]. It is the purpose of this note to extend these results by a weak compactness result for $H^{1}$-functions defined on a periodic manifold satisfying certain bounds (Theorem 4 below). These arise in problems involving fast surface diffusion, cf. $\S 4$. For utilization in the proof of Theorem 4, we also show an extension lemma (Lemma 5), which may be useful in related contexts as well.

We briefly describe the setting and summarize important results required in what follows. Let $\Omega \subset \mathbb{R}^{n}$ be a domain, and further let $\Omega_{\varepsilon}=\bigcup_{k \in \mathbb{Z}^{n}} \varepsilon(k+Y) \cap \Omega$ and $\Gamma_{\varepsilon}=\bigcup_{k \in \mathbb{Z}^{n}} \varepsilon(k+\Gamma) \cap \Omega$ be sets with periodic fine-structure with unit cell $Y=[0,1]^{n}$ and a smooth manifold $\Gamma \subset Y$, such that $\Gamma_{\varepsilon}$ is smooth and connected and $\Omega$ is representable by a finite union of axis-parallel cuboids, each of which is assumed to have corner coordinates in $\mathbb{Q}^{n}$. This last technical assumption is required in order to use a certain extension operator, cf. Remark 6. Note that there also exist recent works in the context of periodic unfolding and manifolds, where the manifold itself is not periodic but has a periodic pattern on its surface [7], which is different from the setting considered here.

Let $\Xi_{\varepsilon}:=\left\{\xi \in \mathbb{Z}^{n} \mid \varepsilon(\xi+Y) \subset \Omega\right\}$ and $\hat{\Omega}_{\varepsilon}:=\operatorname{interior}\left\{\bigcup_{\xi \in \Xi_{\varepsilon}} \varepsilon(\xi+\bar{Y})\right\}$.

For every $z \in \mathbb{R}^{n}$, we define $[z]_{Y}$ as the unique integer combination $\sum_{i=1}^{n} k_{i} e_{i}$ of the periods such that $\{z\}_{Y}=z-[z]_{Y} \in Y$. The periodic unfolding operator $\mathcal{T}_{\varepsilon}$ is then defined as follows [4]:

Email addresses: imgraf@sfu.ca (Isabell Graf), peter@math.uni-augsburg.de (Malte A. Peter) 
Definition 1 Let $\varphi \in L^{p}\left(\Omega_{\varepsilon}\right), p \in[1, \infty]$. For any $\varepsilon>0$ we define $\mathcal{T}_{\varepsilon}: L^{p}\left(\Omega_{\varepsilon}\right) \rightarrow L^{p}(\Omega \times Y)$ such that

$$
\left[\mathcal{T}_{\varepsilon}(\varphi)\right](x, y)=\varphi\left(\varepsilon\left[\frac{x}{\varepsilon}\right]_{Y}+\varepsilon y\right) \text { a.e. for }(x, y) \in \hat{\Omega}_{\varepsilon} \times Y, \quad\left[\mathcal{T}_{\varepsilon}(\varphi)\right](x, y)=0 \text { a.e. for }(x, y) \in \Omega \backslash \hat{\Omega}_{\varepsilon} \times Y \text {. }
$$

The main advantage of using the periodic unfolding operator is that $\mathcal{T}_{\varepsilon}(\varphi)$ is defined on the fixed domain $\Omega \times Y$ even for varying $\varepsilon$. Thus, we may use standard convergence results from functional analysis. For example, the following weak compactness result in $H^{1}$ is proven in [5]. It is the main ingredient in identifying the limit problem when homogenizing typical reaction-diffusion equations stated on $\Omega_{\varepsilon}$.

Theorem 2 For every $\varepsilon>0$, let $\varphi_{\varepsilon}$ be in $H^{1}\left(\Omega_{\varepsilon}\right)$ and let $\left\|\varphi_{\varepsilon}\right\|_{H^{1}\left(\Omega_{\varepsilon}\right)}$ be bounded independently of $\varepsilon$. Then there exists $\varphi \in H^{1}(\Omega)$ and $\hat{\varphi} \in L^{2}\left(\Omega, H_{\text {per }}^{1}(Y)\right)$ such that, up to a subsequence,

$$
\mathcal{T}_{\varepsilon}\left(\varphi_{\varepsilon}\right) \stackrel{\varepsilon \rightarrow 0}{\rightarrow} \varphi \text { weakly in } L^{2}\left(\Omega, H_{\mathrm{per}}^{1}(Y)\right), \quad \quad \mathcal{T}_{\varepsilon}\left(\nabla_{x} \varphi_{\varepsilon}\right) \stackrel{\varepsilon \rightarrow 0}{\rightarrow^{0}} \nabla_{x} \varphi+\nabla_{y} \hat{\varphi} \text { weakly in } L^{2}\left(\Omega, L^{2}(Y)\right)
$$

When internal boundary terms are to be homogenized, e.g. arising from interface conditions or surface concentrations, the boundary periodic unfolding operator $\mathcal{T}_{\varepsilon}^{b}$ is introduced. It is defined as follows, see [6].

Definition 3 Let $\varphi \in L^{p}\left(\Gamma_{\varepsilon}\right), p \in[1, \infty]$. Then the boundary periodic unfolding operator $\mathcal{T}_{\varepsilon}^{b}: L^{p}\left(\Gamma_{\varepsilon}\right) \rightarrow$ $L^{p}(\Omega \times \Gamma)$ is defined as

$$
\mathcal{T}_{\varepsilon}^{b}(\varphi)(x, y)=\varphi\left(\varepsilon\left[\frac{x}{\varepsilon}\right]+\varepsilon y\right) \text { a.e. for }(x, y) \in \hat{\Omega}_{\varepsilon} \times \Gamma, \quad \mathcal{T}_{\varepsilon}^{b}(\varphi)(x, y)=0 \text { a.e. for }(x, y) \in \Omega \backslash \hat{\Omega}_{\varepsilon} \times \Gamma .
$$

It is well-known in periodic homogenization that different scalings with the homogenization parameter lead to different limit behaviour (see e.g. [14], where weak compactness results in the spirit of Theorem 2 are discussed for different scalings). The canonical scaling of surface terms is $\varepsilon$, that of surface gradients is $\varepsilon^{3}$, which is due to the fact that $\left|\Gamma_{\varepsilon}\right| \sim \varepsilon^{-1}$ in the limit. For these scalings, associated with slow diffusion, local (or microscopic) diffusion in the unit cell, i.e. with respect to the $y$-variable, is obtained in the homogenization limit $[1,13]$.

The purpose of this contribution is to extend the results to fast diffusion, associated with a scaling of the surface gradients with $\varepsilon^{1}$. It turns out that this leads to global (or macroscopic) diffusion, i.e. with respect to the $x$-variable, in the homogenization limit.

In what follows, we formulate the main result in $\S 2$, present the proof in $\S 3$ and apply it to homogenize a prototypical diffusion problem in $\S 4$.

2. Statement of the main result. The main result is the following weak compactness result for $H^{1}$ functions defined on a manifold $\Gamma_{\varepsilon}$.

Theorem 4 Let $\varphi_{\varepsilon} \in H^{1}\left(\Gamma_{\varepsilon}\right)$ be a sequence of functions with

$$
\varepsilon\left\|\varphi_{\varepsilon}\right\|_{L^{2}\left(\Gamma_{\varepsilon}\right)}^{2}+\varepsilon\left\|\nabla_{\Gamma} \varphi_{\varepsilon}\right\|_{L^{2}\left(\Gamma_{\varepsilon}\right)}^{2} \leq C,
$$

where $C$ is independent of $\varepsilon$. Let $P_{\Gamma}$ be the orthogonal projection from $\mathbb{R}^{n}$ to the tangent space $T_{y} \Gamma$ for every $y \in \Gamma$. Then two assertions hold true.

1. There exists a function $\varphi_{0} \in H^{1}(\Omega)$ such that, up to a subsequence,

$$
\mathcal{T}_{\varepsilon}^{b}\left(\varphi_{\varepsilon}\right) \stackrel{\varepsilon \rightarrow 0}{\rightarrow} \varphi_{0} \text { weakly in } L^{2}(\Omega \times \Gamma) \text { and } \varepsilon \int_{\Gamma_{\varepsilon}} \varphi_{\varepsilon} \psi d \sigma_{x} \stackrel{\varepsilon \rightarrow 0}{\rightarrow} \frac{|\Gamma|}{|Y|} \int_{\Omega} \varphi_{0} \psi d x \text { for all } \psi \in C^{\infty}(\Omega)
$$

2. There exists a $\hat{\varphi} \in L^{2}\left(\Omega, H_{\mathrm{per}}^{1}(\Gamma)\right)$ such that, up to a subsequence,

$$
\mathcal{T}_{\varepsilon}^{b}\left(\nabla_{x} \varphi_{\varepsilon}\right) \stackrel{\varepsilon \rightarrow 0}{\longrightarrow} P_{\Gamma} \nabla_{x} \varphi_{0}+\nabla_{\Gamma} \hat{\varphi} \text { weakly in } L^{2}(\Omega \times \Gamma)
$$

3. Proof of the main result. For later use in the proof of Theorem 4, we first show an inverse trace lemma.

Lemma 5 Let $\Omega \subset \mathbb{R}^{n}$ and $\Gamma \subset[0,1]^{n}=Y$ be a smooth and compact hypersurface such that $\Gamma_{\varepsilon}=$ $\bigcup_{k \in \mathbb{Z}^{n}} \varepsilon(k+\Gamma) \cap \Omega$ is a smooth, periodic and connected hypersurface. Let $f_{\varepsilon} \in H^{1}\left(\Gamma_{\varepsilon}\right)$. Then, there exists a function $u_{\varepsilon} \in H^{1}(\Omega)$ with $u_{\left.\varepsilon\right|_{\Gamma_{\varepsilon}}}=f_{\varepsilon}$ such that

$$
\left\|u_{\varepsilon}\right\|_{L^{2}(\Omega)}^{2} \leq C_{1} \varepsilon\left\|f_{\varepsilon}\right\|_{L^{2}\left(\Gamma_{\varepsilon}\right)}^{2} \quad \text { and } \quad\left\|\nabla u_{\varepsilon}\right\|_{L^{2}(\Omega)}^{2} \leq C_{2} \varepsilon\left(\left\|f_{\varepsilon}\right\|_{L^{2}\left(\Gamma_{\varepsilon}\right)}^{2}+\left\|\nabla_{\Gamma} f_{\varepsilon}\right\|_{L^{2}\left(\Gamma_{\varepsilon}\right)}^{2}\right)
$$

for constants $C_{1}, C_{2}>0$ independent of $\varepsilon$. 
Proof Because $\Gamma$ is smooth and compact, the normal in each point $y \in \Gamma, n_{y}$, is well-defined. For small $\delta>0$ we define $Y^{*}=\left\{y+d n_{y} \mid y \in \Gamma, d \in(-\delta, \delta)\right\}$ so that for every $z \in Y^{*}$ there exist unique $y \in \Gamma$ and $d \in(-\delta, \delta)$ with $y+d n_{y}=z$. On the tube $Y^{*}$ we define a Riemannian metric by $g_{i j}, i, j=1, \ldots n$, such that the tangential vectors $\frac{\mathrm{d}}{\mathrm{d} y^{i}}, i=1, \ldots, n-1$ form a basis of the tangent space $T_{y} \Gamma$ and $\frac{\mathrm{d}}{\mathrm{d} y^{n}}$ treats the normal direction $n_{y}$. Because $n_{y}$ is orthogonal to the tangent space $T_{y} \Gamma$, it follows $g_{i n}=g_{n i}=g^{i n}=g^{n i}=0$ for $i=1, \ldots, n-1$.

Now we define $\Omega_{\varepsilon}^{*}=\bigcup_{k \in \mathbb{Z}^{n}} \varepsilon\left(k+Y^{*}\right) \cap \Omega$ and consider the scaled unit cell $\varepsilon Y$ with scaled tube $\varepsilon Y^{*}$. The width of $\varepsilon Y^{*}$ is now $2 \delta \varepsilon$ and $d \in(-\varepsilon \delta, \varepsilon \delta)$. Analogously one finds for every $x \in \Omega_{\varepsilon}^{*}$ unique $y \in \Gamma_{\varepsilon}$ and $d \in(-\varepsilon \delta, \varepsilon \delta)$ such that $y+d n_{y}=x$. Because the additional direction $n_{y}$ is perpendicular to the tangent space of $\Gamma_{\varepsilon}$, it holds that $\left|\Omega_{\varepsilon}^{*}\right| \leq 2 \varepsilon \delta c_{1}\left|\Gamma_{\varepsilon}\right|$ for a constant $c_{1}>0$ independent of $\varepsilon$, which can be seen by calculating the Lebesgue-measure of the manifold $\Gamma_{\varepsilon}$ using its charts by means of integration by substitution.

We define a function $\tilde{u}_{\varepsilon} \in H^{1}\left(\Omega_{\varepsilon}^{*}\right)$ by $\tilde{u}_{\varepsilon}(x)=\tilde{u}_{\varepsilon}\left(y+d n_{y}\right)=f_{\varepsilon}(y)$ for every $x \in \Omega_{\varepsilon}^{*}$. Then, the following holds:

$$
\left\|\tilde{u}_{\varepsilon}\right\|_{L^{2}\left(\Omega_{\varepsilon}^{*}\right)}^{2}=\int_{\Omega_{\varepsilon}^{*}} \tilde{u}_{\varepsilon}^{2}\left(y+d n_{y}\right) \mathrm{d} x \leq 2 c_{1} \varepsilon \delta \int_{\Gamma_{\varepsilon}} f_{\varepsilon}^{2}(y) \mathrm{d} \sigma_{y}=2 c_{1} \delta \varepsilon\left\|f_{\varepsilon}\right\|_{L^{2}\left(\Gamma_{\varepsilon}\right)}^{2} .
$$

To estimate the gradient, we consider the gradient in the coordinates $\frac{\mathrm{d}}{\mathrm{d} y^{i, \varepsilon}}, i=1, \ldots, n-1$ on $T_{y} \Gamma_{\varepsilon}$, and exploit that $f_{\varepsilon}(y)$ is independent of $d$,

$$
\nabla_{x} \tilde{u}_{\varepsilon}(x)=\sum_{i j=1}^{n} g^{i j, \varepsilon}(x) \frac{\partial \tilde{u}_{\varepsilon}}{\partial y^{j, \varepsilon}}\left(y+d n_{y}\right) \frac{\mathrm{d}}{\mathrm{d} y^{i, \varepsilon}}=\sum_{i j=1}^{n} g^{i j, \varepsilon}(x) \frac{\partial f_{\varepsilon}}{\partial y^{j, \varepsilon}}(y) \frac{\mathrm{d}}{\mathrm{d} y^{i, \varepsilon}}=\sum_{i j=1}^{n-1} g^{i j, \varepsilon}(x) \frac{\partial f_{\varepsilon}}{\partial y^{j, \varepsilon}}(y) \frac{\mathrm{d}}{\mathrm{d} y^{i, \varepsilon}}
$$

for every $x \in \Omega_{\varepsilon}^{*}$ with $x=y+d n_{y}, y \in \Gamma_{\varepsilon}$. Since the Riemannian metric tensor $g^{i j, \varepsilon}$ is continuous and $\Gamma_{\varepsilon}$ compact, there exists a constant $c_{2}$ such that for small $\delta>0$

$$
\left|\nabla_{x} \tilde{u}_{\varepsilon}(x)\right|^{2} \leq c_{2}\left|\sum_{i j=1}^{n-1} g^{i j, \varepsilon}(y) \frac{\partial f_{\varepsilon}}{\partial y^{j, \varepsilon}}(y) \frac{\mathrm{d}}{\mathrm{d} y^{i, \varepsilon}}\right|^{2}=c_{2}\left|\nabla_{\Gamma} f_{\varepsilon}(y)\right|^{2}
$$

Now the norm of the gradient $u_{\varepsilon}$ can be estimated,

$$
\left\|\nabla \tilde{u}_{\varepsilon}\right\|_{L^{2}\left(\Omega_{\varepsilon}^{*}\right)}^{2} \leq c_{2} \int_{\Omega_{\varepsilon}^{*}}\left|\nabla_{\Gamma} f_{\varepsilon}(y)\right|^{2} \mathrm{~d} x \leq c_{1} c_{2} 2 \varepsilon \delta \int_{\Gamma_{\varepsilon}}\left|\nabla_{\Gamma} f_{\varepsilon}(y)\right|^{2} \mathrm{~d} y=2 \delta \varepsilon c_{1} c_{2}\left\|\nabla_{\Gamma} f_{\varepsilon}\right\|_{L^{2}\left(\Gamma_{\varepsilon}\right)}^{2} .
$$

Therefore, we constructed an extension from $\Gamma_{\varepsilon}$ to $\Omega_{\varepsilon}^{*}$ satisfying the estimates claimed. We continue by extending from $\Omega_{\varepsilon}^{*}$ to $\Omega$ by using the extension operator from the article [10] for connected sets $\Omega_{\varepsilon}$, which leads to an extended function $u_{\varepsilon} \in H^{1}(\Omega)$ such that

$$
\left\|u_{\varepsilon}\right\|_{L^{2}(\Omega)}^{2} \leq C\left\|\tilde{u}_{\varepsilon}\right\|_{L^{2}\left(\Omega_{\varepsilon}^{*}\right)}^{2} \quad \text { and } \quad\left\|\nabla u_{\varepsilon}\right\|_{L^{2}(\Omega)}^{2} \leq C\left(\left\|\tilde{u}_{\varepsilon}\right\|_{L^{2}\left(\Omega_{\varepsilon}^{*}\right)}^{2}+\left\|\nabla \tilde{u}_{\varepsilon}\right\|_{L^{2}\left(\Omega_{\varepsilon}^{*}\right)}^{2}\right) .
$$

This completes the proof with the constants $C_{1}=2 c_{1} \delta C$ and $C_{2}=2 \delta c_{1} C \max \left\{1, c_{2}\right\}$.

Remark 6 The statement of Lemma 5 can be strengthened slightly to the separate estimates $\left\|u_{\varepsilon}\right\|_{L^{2}(\Omega)}^{2} \leq C_{1} \varepsilon\left\|f_{\varepsilon}\right\|_{L^{2}\left(\Gamma_{\varepsilon}\right)}^{2}$ and $\left\|\nabla u_{\varepsilon}\right\|_{L^{2}(\Omega)}^{2} \leq C_{2} \varepsilon\left\|\nabla_{\Gamma} f_{\varepsilon}\right\|_{L^{2}\left(\Gamma_{\varepsilon}\right)}^{2}$, if an extension operator from $H^{1}\left(\Omega_{\varepsilon}^{*}\right)$ to $H^{1}(\Omega)$ with separate estimates $\left\|u_{\varepsilon}\right\|_{L^{2}(\Omega)}^{2} \leq C\left\|\tilde{u}_{\varepsilon}\right\|_{L^{2}\left(\Omega_{\varepsilon}^{*}\right)}^{2}$ and $\left\|\nabla u_{\varepsilon}\right\|_{L^{2}(\Omega)}^{2} \leq C\left\|\nabla \tilde{u}_{\varepsilon}\right\|_{L^{2}\left(\Omega_{\varepsilon}^{*}\right)}^{2}$ is available. For example, the extension operator described in [12] can be used in this way if the function $u_{\varepsilon}$ vanishes at the exterior boundary of $\Omega_{\varepsilon}^{*}$ and, in this case, the technical assumption on the domain $\Omega$ being representable by cuboids can be dropped as well. We refer to [3] and [10] for further discussions on boundary behaviour and extensions in this context.

\section{Proof of Theorem 4}

1. We use Lemma 5 to deduce the existence of a function $\tilde{\varphi}_{\varepsilon} \in H^{1}(\Omega)$ such that $\gamma\left(\varphi_{\varepsilon}\right)=\left.\tilde{\varphi}_{\varepsilon}\right|_{\Gamma_{\varepsilon}}=\varphi_{\varepsilon}$ and

$$
\left\|\tilde{\varphi}_{\varepsilon}\right\|_{L^{2}(\Omega)}+\left\|\nabla \tilde{\varphi}_{\varepsilon}\right\|_{L^{2}(\Omega)}^{2} \leq c_{1} \varepsilon\left(\left\|\varphi_{\varepsilon}\right\|_{L^{2}\left(\Gamma_{\varepsilon}\right)}^{2}+\left\|\nabla_{\Gamma} \varphi_{\varepsilon}\right\|_{L^{2}\left(\Gamma_{\varepsilon}\right)}^{2}\right) \leq C .
$$

Hence, $\tilde{\varphi}_{\varepsilon}$ has a weak limit function $\varphi_{0}$ in $H^{1}(\Omega)$. We calculate with Theorem 2

$$
\int_{\Omega \times Y} \mathcal{T}_{\varepsilon}\left(\tilde{\varphi}_{\varepsilon}\right)(x, y) \psi(x, y) \mathrm{d} x \mathrm{~d} y \stackrel{\varepsilon \rightarrow 0}{\longrightarrow} \int_{\Omega \times Y} \varphi_{0}(x) \psi(x, y) \mathrm{d} y \mathrm{~d} x
$$


weakly in $L^{2}\left(\Omega, H_{\mathrm{per}}^{1}(Y)\right)$ for all $\psi \in C^{\infty}(\Omega \times Y)$.

The trace operator $\gamma_{\Omega \times \Gamma}: L^{2}\left(\Omega, H^{1}(Y)\right) \rightarrow L^{2}\left(\Omega, L^{2}(\Gamma)\right)$ defined by $\gamma_{\Omega \times \Gamma}(\varphi)=\varphi_{\left.\right|_{\Omega \times \Gamma}}$ commutes with $\mathcal{T}_{\varepsilon}$ as follows. Let $\psi \in H^{1}\left(\Omega_{\varepsilon}\right)$, then

$$
\mathcal{T}_{\varepsilon}^{b}(\gamma(\psi))(x, y)=\mathcal{T}_{\varepsilon}^{b}\left(\psi_{\Gamma_{\varepsilon}}\right)(x, y)=\psi(\varepsilon\left[\frac{x}{\varepsilon}\right]_{Y}+\varepsilon \underbrace{y}_{\in \Gamma})=\mathcal{T}_{\varepsilon}(\psi)_{\mid \Omega \times \Gamma}(x, y)=\gamma_{\Omega \times \Gamma}\left(\mathcal{T}_{\varepsilon}(\psi)\right)(x, y) .
$$

It holds that $\mathcal{T}_{\varepsilon}\left(\tilde{\varphi}_{\varepsilon}\right)$ converges weakly in $H_{\text {per }}^{1}(Y)$ in its second variable and the trace operator is linear and continuous. Using (1) it follows that

$$
\begin{aligned}
& |Y| \varepsilon \int_{\Gamma_{\varepsilon}} \varphi_{\varepsilon} \psi \mathrm{d} \sigma_{x}=\int_{\Omega \times \Gamma} \mathcal{T}_{\varepsilon}^{b}\left(\varphi_{\varepsilon}\right)(x, y) \mathcal{T}_{\varepsilon}^{b}(\psi)(x, y) \mathrm{d} \sigma_{y} \mathrm{~d} x=\int_{\Omega \times \Gamma} \mathcal{T}_{\varepsilon}^{b}\left(\gamma\left(\tilde{\varphi}_{\varepsilon}\right)\right)(x, y) \mathcal{T}_{\varepsilon}^{b}(\psi)(x, y) \mathrm{d} \sigma_{y} \mathrm{~d} x \\
& \quad=\int_{\Omega \times \Gamma} \gamma_{\Omega \times \Gamma}\left(\mathcal{T}_{\varepsilon}\left(\tilde{\varphi}_{\varepsilon}\right)\right)(x, y) \mathcal{T}_{\varepsilon}^{b}(\psi)(x, y) \mathrm{d} \sigma_{y} \mathrm{~d} x \stackrel{\varepsilon \rightarrow 0}{\rightarrow} \int_{\Omega \times \Gamma} \gamma_{\Omega \times \Gamma}\left(\varphi_{0}\right)(x, y) \psi(x) \mathrm{d} \sigma_{y} \mathrm{~d} x=|\Gamma| \int_{\Omega} \varphi_{0} \psi \mathrm{d} x
\end{aligned}
$$

for all $\psi \in C^{\infty}(\Omega)$. We used the integration formula of the operator $\mathcal{T}_{\varepsilon}^{b}$ in the first step and exploited that $\varphi_{0}$ is independent of $y$ in the last step. This completes the proof of part 1 .

2. To prove the second part of the theorem, we need some additional definitions and properties. We define for every $a \in \mathbb{R}^{n}$ the function $z_{a}: \Gamma \rightarrow \mathbb{R}$ by $z_{a}(y)=a^{T} \cdot y$ and its gradient by using the directional derivatives on $\Gamma$ by $\left\langle\nabla_{\Gamma} z_{a}, v\right\rangle=d z_{a}(v)=\left.\frac{\mathrm{d}}{\mathrm{d} t}\right|_{t=0} z_{a}(\gamma(t))$ for every $y \in \Gamma$ and for $\gamma:(-\delta, \delta) \rightarrow \Gamma, \gamma(0)=y$ and $\dot{\gamma}(0)=v$. We find that $\left.\frac{\mathrm{d}}{\mathrm{d} t}\right|_{t=0} z_{a}(\gamma(t))=\left.\frac{\mathrm{d}}{\mathrm{d} t}\right|_{t=0} a^{T} \cdot \gamma(t)=a^{T} \cdot \dot{\gamma}(t)=a^{T} \cdot v$ for all $v \in T_{y} \Gamma$. The only element of $T_{y} \Gamma$ satisfying $\left\langle\nabla_{\Gamma} z_{a}, v\right\rangle=a^{T} \cdot v$ for all $v \in T_{y} \Gamma$ is the orthogonal projection of $a$ to $T_{y} \Gamma$. Hence, $\nabla_{\Gamma} z_{a}=P_{\Gamma} a$. We define $z_{a}^{c}(y)=z_{a}(y)-\frac{1}{|\Gamma|} \int_{\Gamma} z_{a}(y) \mathrm{d} \sigma_{y}$. Then for any vector $a \in \mathbb{R}^{n}$ it is true that

$$
\int_{\Gamma} z_{a}^{c}(y) \mathrm{d} \sigma_{y}=\int_{\Gamma} z_{a}(y) \mathrm{d} \sigma_{y}-\int_{\Gamma} \frac{1}{|\Gamma|} \int_{\Gamma} z_{a}(y) \mathrm{d} \sigma_{y} \mathrm{~d} \sigma_{y}=\int_{\Gamma} z_{a}(y) \mathrm{d} \sigma_{y}-\int_{\Gamma} z_{a}(y) \mathrm{d} \sigma_{y}=0 .
$$

Further we define $M_{\varepsilon}^{b}\left(\varphi_{\varepsilon}\right)=\frac{1}{|\Gamma|} \int_{\Gamma} \mathcal{T}_{\varepsilon}^{b}\left(\varphi_{\varepsilon}\right)(x, y) \mathrm{d} \sigma_{y}$ and $Z_{\varepsilon}^{b}=\frac{1}{\varepsilon}\left(\mathcal{T}_{\varepsilon}^{b}\left(\varphi_{\varepsilon}\right)-M_{\varepsilon}^{b}\left(\varphi_{\varepsilon}\right)\right)$. Then we deduce $\int_{\Gamma} Z_{\varepsilon}^{b} \mathrm{~d} \sigma_{y}=0$ and $\nabla_{\Gamma} Z_{\varepsilon}^{b}=\frac{1}{\varepsilon} \nabla_{\Gamma} \mathcal{T}_{\varepsilon}^{b}\left(\varphi_{\varepsilon}\right)=\mathcal{T}_{\varepsilon}^{b}\left(\nabla_{x} \varphi_{\varepsilon}\right)$, since $M_{\varepsilon}^{b}\left(\varphi_{\varepsilon}\right)$ is independent of $y$.

Having finished these preparations, we now come to the main part of the proof of part 2. We consider $\left\|Z_{\varepsilon}^{b}\left(\varphi_{\varepsilon}\right)-z_{\nabla_{x} \varphi_{0}}^{c}\right\|_{L^{2}(\Gamma \times \Omega)}^{2}$. Note that for the $y$-component, the $x$-gradient $\nabla_{x} \varphi_{0}$ looks like a vector in $\mathbb{R}^{n}$ because $\varphi_{0}$ is independent of $y$. We use the Poincaré inequality on Riemannian manifolds (cf. [11]) to obtain

$$
\begin{aligned}
\| Z_{\varepsilon}^{b}\left(\varphi_{\varepsilon}\right)- & z_{\nabla_{x} \varphi_{0}}^{c}\left\|_{L^{2}(\Omega \times \Gamma)}^{2} \leq \int_{\Omega} C\right\| \nabla_{\Gamma} Z_{\varepsilon}^{b}\left(\varphi_{\varepsilon}\right)-\nabla_{\Gamma} z_{\nabla_{x} \varphi_{0}}^{c} \|_{L^{2}(\Gamma)}^{2} \mathrm{~d} x \\
\quad & C \int_{\Omega}\left\|\mathcal{T}_{\varepsilon}^{b}\left(\nabla_{x} \varphi_{\varepsilon}\right)-P_{\Gamma} \nabla_{x} \varphi_{0}\right\|_{L^{2}(\Gamma)}^{2} \mathrm{~d} x \leq C \varepsilon|Y|\left\|\nabla_{x} \varphi_{\varepsilon}\right\|_{L^{2}\left(\Gamma_{\varepsilon}\right)}^{2}+C|\Gamma| \underbrace{\left\|P_{\Gamma}\right\|_{2}}_{\leq 1}\left\|\nabla_{x} \varphi_{0}\right\|_{L^{2}(\Omega)}^{2} \leq \tilde{C}
\end{aligned}
$$

for a constant $\tilde{C}>0$ independent of $\varepsilon$. Hence, $Z_{\varepsilon}^{b}\left(\varphi_{\varepsilon}\right)-z_{\nabla_{x} \varphi_{0}}^{c}$ converges weakly to a function $\hat{\varphi} \in$ $L^{2}\left(\Omega, H^{1}(\Gamma)\right)$, up to a subsequence, i.e. for $\varepsilon \rightarrow 0$

$$
Z_{\varepsilon}^{b}\left(\varphi_{\varepsilon}\right) \rightarrow \hat{\varphi}+z_{\nabla_{x} \varphi_{0}}^{c}, \quad \mathcal{T}_{\varepsilon}^{b}\left(\nabla_{x} \varphi_{\varepsilon}\right)=\nabla_{\Gamma} Z_{\varepsilon}^{b}\left(\varphi_{\varepsilon}\right) \rightarrow \nabla_{\Gamma} \hat{\varphi}+P_{\Gamma} \nabla_{x} \varphi_{0} .
$$

Finally, we need to show that $\hat{\varphi} \in L^{2}\left(\Omega, H_{\mathrm{per}}^{1}(\Gamma)\right)$, this means that $\hat{\varphi}$ is $Y$-periodic in its second argument. For this purpose, we define $\partial_{i} Y:=\left\{y \in Y \mid y_{i}=0\right\}$ for $i=1, \ldots, n$. We extend the functions from $\Omega$ to $\mathbb{R}^{n}$ by zero and compute

$$
\begin{aligned}
\int_{\mathbb{R}^{n}} \int_{\Gamma \cap \partial_{i} Y}\left[Z_{\varepsilon}^{b}\left(\varphi_{\varepsilon}\right)\left(x, y+e_{i}\right)-Z_{\varepsilon}^{b}\left(\varphi_{\varepsilon}\right)(x, y)\right] \psi(x, y) \mathrm{d} \sigma_{y} \mathrm{~d} x \\
=\int_{\mathbb{R}^{n}} \int_{\Gamma \cap \partial_{i} Y} \frac{1}{\varepsilon}\left[\mathcal{T}_{\varepsilon}^{b}\left(\varphi_{\varepsilon}\right)\left(x, y+e_{i}\right)-\mathcal{T}_{\varepsilon}^{b}\left(\varphi_{\varepsilon}\right)(x, y)\right] \psi(x, y) \mathrm{d} \sigma_{y} \mathrm{~d} x \\
=\int_{\mathbb{R}^{n}} \int_{\Gamma \cap \partial_{i} Y} \frac{1}{\varepsilon} \mathcal{T}_{\varepsilon}^{b}\left(\varphi_{\varepsilon}\right)(x, y) \psi\left(x-\varepsilon e_{i}, y\right)-\frac{1}{\varepsilon} \mathcal{T}_{\varepsilon}^{b}\left(\varphi_{\varepsilon}\right)(x, y) \psi(x, y) \mathrm{d} \sigma_{y} \mathrm{~d} x
\end{aligned}
$$




$$
\begin{aligned}
& =\int_{\mathbb{R}^{n}} \int_{\Gamma \cap \partial_{i} Y} \mathcal{T}_{\varepsilon}^{b}\left(\varphi_{\varepsilon}\right)(x, y) \frac{\psi\left(x-\varepsilon e_{i}, y\right)-\psi(x, y)}{\varepsilon} \mathrm{d} \sigma_{y} \mathrm{~d} x \\
& \stackrel{\varepsilon \rightarrow 0}{\rightarrow} \int_{\mathbb{R}^{n}} \int_{\Gamma \cap \partial_{i} Y} \gamma\left(\varphi_{0}\right)\left(-\frac{d \psi}{\mathrm{d} x_{i}}\right) \mathrm{d} \sigma_{y} \mathrm{~d} x=\int_{\mathbb{R}^{n}} \int_{\Gamma \cap \partial_{i} Y} \frac{d \varphi_{0}}{\mathrm{~d} x_{i}} \psi \mathrm{d} \sigma_{y} \mathrm{~d} x=\int_{\mathbb{R}^{n}} \int_{\Gamma \cap \partial_{i} Y} e_{i}^{T} \cdot \nabla_{x} \varphi_{0} \psi \mathrm{d} \sigma_{y} \mathrm{~d} x
\end{aligned}
$$

for all $\psi \in C^{\infty}\left(\mathbb{R}^{n} \times \Gamma\right)$. Because of the extension by zero on $\mathbb{R}^{n}$ we conclude for the domain $\Omega$

$$
\int_{\Omega} \int_{\Gamma \cap \partial_{i} Y}\left[Z_{\varepsilon}^{b}\left(\varphi_{\varepsilon}\right)\left(x, y+e_{i}\right)-Z_{\varepsilon}^{b}\left(\varphi_{\varepsilon}\right)(x, y)\right] \psi(x, y) \mathrm{d} \sigma_{y} \mathrm{~d} x \stackrel{\varepsilon \rightarrow 0}{\rightarrow} \int_{\Omega} \int_{\Gamma \cap \partial_{i} Y} e_{i}^{T} \cdot \nabla_{x} \varphi_{0} \psi \mathrm{d} \sigma_{y} \mathrm{~d} x .
$$

On the other hand we calculate

$$
\begin{aligned}
\int_{\Omega} \int_{\Gamma \cap \partial_{i} Y}\left[z_{\nabla_{x} \varphi_{0}}^{c}\left(y+e_{i}\right)\right. & \left.-z_{\nabla_{x} \varphi_{0}}^{c}(y)\right] \psi(x, y) \mathrm{d} \sigma_{y} \mathrm{~d} x \\
= & \int_{\Omega} \int_{\Gamma \cap \partial_{i} Y}\left[\nabla_{x} \varphi_{0} \cdot\left(y+e_{i}\right)-\nabla_{x} \varphi_{0} \cdot y\right] \psi \mathrm{d} \sigma_{y} \mathrm{~d} x=\int_{\Omega} \int_{\Gamma \cap \partial_{i} Y} e_{i}^{T} \cdot \nabla_{x} \varphi_{0} \psi \mathrm{d} \sigma_{y} \mathrm{~d} x
\end{aligned}
$$

for all $\psi \in C^{\infty}(\Omega \times \Gamma)$. With $Z_{\varepsilon}^{b}\left(\varphi_{\varepsilon}\right) \rightarrow \hat{\varphi}+z_{\nabla_{x} \varphi_{0}}^{c}$ we conclude that

$$
\int_{\Omega} \int_{\Gamma \times \partial_{i} Y}\left[\hat{\varphi}\left(x, y+e_{i}\right)-\hat{\varphi}(x, y)\right] \psi(x, y) \mathrm{d} \sigma_{y} \mathrm{~d} x=0
$$

for all $\psi \in C^{\infty}(\Omega \times \Gamma)$ and $i=1, \ldots, n$. So $\hat{\varphi} \in L^{2}\left(\Omega, H_{\mathrm{per}}^{1}(\Gamma)\right)$ and the proof is completed.

4. Example. Theorem 4 can be used to derive global diffusion on a manifold for a partial differential equation in the homogenization limit. For example, this arises in biomedical applications, where molecules diffuse fast on the surface of fine-structured membranes in human cells, cf. [8, 9] and references therein. The following example illustrates how Theorem 4 can be used in this context.

Let $\Gamma \subset[0,1]^{n}$ be a smooth, $n-1$ dimensional manifold, such that $\Gamma_{\varepsilon}=\bigcup_{k \in \mathbb{Z}^{n}} \varepsilon(k+\Gamma)$ is periodic, connected and smooth. Let $\Omega \subset \mathbb{R}^{n}$ be bounded, $f \in C\left(\Omega, C_{\text {per }}(Y)\right)$ with $f_{\varepsilon}(x):=f\left(x, \frac{x}{\varepsilon}\right)$, and $D_{\varepsilon}(x)=$ $D\left(x, \frac{x}{\varepsilon}\right)$ be an elliptic diffusion tensor on the tangent space of $T_{y} \Gamma_{\varepsilon}$, which is $\varepsilon$-periodic in its second argument and with $\lim _{\varepsilon \rightarrow 0}\left\|D_{\varepsilon}\right\|_{L^{2}\left(\Omega_{\varepsilon}\right)}^{2}=\|D\|_{L^{2}(\Omega \times \Gamma)}^{2}$ bounded. Further, for given $\varepsilon>0$, let $u_{\varepsilon}$ be the solution of the problem

$$
\begin{aligned}
& \partial_{t} u_{\varepsilon}(x, t)-\nabla_{\Gamma} \cdot\left(D_{\varepsilon}(x) \nabla_{\Gamma} u_{\varepsilon}(x, t)\right)+u_{\varepsilon}(x, t)=f_{\varepsilon}(x, t) \quad \text { on } \Gamma_{\varepsilon} \text {, } \\
& u_{\varepsilon}(x, t)=0 \quad \text { on } \partial \Omega \cap \Gamma_{\varepsilon} \text {. }
\end{aligned}
$$

We multiply the weak formulation with $\varepsilon$ and find with standard estimations that

$$
\varepsilon\left\|u_{\varepsilon}\right\|_{L^{2}\left(\Gamma_{\varepsilon}\right)}^{2}+\varepsilon\left\|\sqrt{D_{\varepsilon}} \nabla_{\Gamma} u_{\varepsilon}\right\|_{L^{2}\left([0, t] \times \Gamma_{\varepsilon}\right)}^{2}+\varepsilon\left\|u_{\varepsilon}\right\|_{L^{2}\left([0, t] \times \Gamma_{\varepsilon}\right)}^{2} \leq C .
$$

Therefore, the conditions to use Theorem 4 are satisfied. Application of the boundary unfolding operator to the weak formulation, where the test function is denoted by $\psi_{\varepsilon}$, leads to

$$
\begin{array}{r}
\int_{\Omega} \int_{\Gamma} \partial_{t} \mathcal{T}_{\varepsilon}^{b}\left(u_{\varepsilon}\right)(x, y, t) \mathcal{T}_{\varepsilon}^{b}\left(\psi_{\varepsilon}\right)(x, y) \mathrm{d} \sigma_{y} \mathrm{~d} x+\int_{\Omega} \int_{\Gamma} \mathcal{T}_{\varepsilon}^{b}\left(D_{\varepsilon}\right)(x, y) \mathcal{T}_{\varepsilon}^{b}\left(\nabla_{\Gamma} u_{\varepsilon}\right)(x, y, t) \mathcal{T}_{\varepsilon}^{b}\left(\nabla_{\Gamma} \psi_{\varepsilon}\right)(x, y) \mathrm{d} \sigma_{y} \mathrm{~d} x \\
+\int_{\Omega} \int_{\Gamma} \mathcal{T}_{\varepsilon}^{b}\left(u_{\varepsilon}\right)(x, y, t) \mathcal{T}_{\varepsilon}^{b}\left(\psi_{\varepsilon}\right)(x, y) \mathrm{d} \sigma_{y} \mathrm{~d} x=\int_{\Omega} \int_{\Gamma} \mathcal{T}_{\varepsilon}^{b}\left(f_{\varepsilon}\right)(x, y, t) \mathcal{T}_{\varepsilon}^{b}\left(\psi_{\varepsilon}\right)(x, y) \mathrm{d} \sigma_{y} \mathrm{~d} x
\end{array}
$$

Now we use Theorem 4 and find for $\varepsilon \rightarrow 0$, noting that $|Y|=1$,

$$
\begin{array}{r}
|\Gamma| \int_{\Omega} \partial_{t} u_{0}(x, t) \psi_{0}(x) \mathrm{d} x+\int_{\Omega} \int_{\Gamma} D(x, y)\left[\nabla_{\Gamma} \hat{u}(x, y, t)+P_{\Gamma} \nabla_{x} u_{0}(x)\right]\left[\nabla_{\Gamma} \hat{\psi}(x, y)+P_{\Gamma} \nabla_{x} \psi_{0}(x)\right] \mathrm{d} \sigma_{y} \mathrm{~d} x \\
+|\Gamma| \int_{\Omega} u_{0}(x, t) \psi_{0}(x) \mathrm{d} x=\int_{\Omega} \int_{\Gamma} f_{0}(x, y, t) \psi_{0}(x) \mathrm{d} \sigma_{y} \mathrm{~d} x .
\end{array}
$$

To determine the cell problem we first set $\psi_{0}=0$. Let $\hat{u}(x, y, t)=\sum_{i=1}^{n} \partial_{x_{i}} u_{0}(x) \chi_{i}(y, t)$ and $\nabla_{\Gamma} \hat{u}(x, y, t)=$ $\sum_{i=1}^{n} \partial_{x_{i}} u_{0}(x) \nabla_{\Gamma} \chi_{i}(y, t)$ for some $\chi_{i}(y, t): \Gamma \times[0, T] \rightarrow \mathbb{R}, i=1, \ldots, n$. Further, we write $P_{\Gamma} \nabla_{x} u_{0}$ as 
$\sum_{i=1}^{n} \partial_{x_{i}} u_{0} P_{\Gamma} e_{i}$ and obtain

$$
\begin{aligned}
\int_{\Omega} \int_{\Gamma} D(x, y)\left[\sum_{i=1}^{n} \partial_{x_{i}} u_{0} \nabla_{\Gamma} \chi_{i}(y, t)+\right. & \left.\sum_{i=1}^{n} \partial_{x_{i}} u_{0} P_{\Gamma} e_{i}\right] \nabla_{\Gamma} \hat{\psi}(y, t) \mathrm{d} \sigma_{y} \mathrm{~d} x \\
& =\int_{\Omega} \sum_{i=1}^{n} \partial_{x_{i}} u_{0} \int_{\Gamma} D(x, y)\left(\nabla_{\Gamma} \chi_{i}(y, t)+P_{\Gamma} e_{i}\right) \nabla_{\Gamma} \hat{\psi}(x, y) \mathrm{d} \sigma_{y} \mathrm{~d} x=0
\end{aligned}
$$

for all $\hat{\psi} \in C^{\infty}\left(\Omega, C_{\mathrm{per}}^{\infty}(\Gamma)\right)$. Hence, the strong formulation of the cell problem is given by

$$
\begin{aligned}
-\nabla_{\Gamma} \cdot D(x, y)\left(\nabla_{\Gamma} \chi_{i}(y, t)+P_{\Gamma} e_{i}\right) & =0 & & \text { in } \Gamma, \\
D(x, y)\left(\nabla_{\Gamma} \chi_{i}(y, t)+P_{\Gamma} e_{i}\right) \cdot n & =0 & & \text { on } \partial \Gamma,
\end{aligned}
$$

and $\chi_{i} Y$-periodic for all $i=1, \ldots, n$. This equation is well-defined since $D$ maps elements of the tangent space $T_{y} \Gamma$ into the tangent space $T_{y} \Gamma$.

Now, we set $\hat{\psi}=0$. Then, after a brief computation, we obtain

$$
\begin{array}{r}
|\Gamma| \int_{\Omega} \partial_{t} u_{0}(x, t) \psi_{0}(x) \mathrm{d} x+\int_{\Omega} \sum_{i, j=1}^{n} \partial_{x_{i}} u_{0} \int_{\Gamma}\left(P_{\Gamma}^{T} D(x, y)\left(\nabla_{\Gamma} \chi_{i}(y, t)+P_{\Gamma} e_{i}\right)\right)_{j} \mathrm{~d} \sigma_{y} \partial_{x_{j}} \psi_{0} \mathrm{~d} x \\
\quad+|\Gamma| \int_{\Omega} u_{0}(x, t) \psi_{0}(x) \mathrm{d} x=\int_{\Omega} \int_{\Gamma} f_{0}(x, y, t) \psi_{0}(x) \mathrm{d} \sigma_{y} \mathrm{~d} x,
\end{array}
$$

for all $\psi_{0} \in C^{\infty}(\Omega)$. Since the orthogonal projection is symmetric $P_{\Gamma}^{T}=P_{\Gamma}$ and $D(x, y)\left(\nabla_{\Gamma} \chi_{i}(y, t)+P_{\Gamma} e_{i}\right)$ is already in the tangent space $T_{y} \Gamma$, we drop the first $P_{\Gamma}$. Defining

$$
s_{i j}(x)=\int_{\Gamma}\left(D(x, y)\left(\nabla_{\Gamma} \chi_{j}(y, t)+P_{\Gamma} e_{j}\right)\right)_{i} \mathrm{~d} \sigma_{y}, \quad S=\left(s_{i j}\right)_{i, j=1, \ldots, n},
$$

we find the strong formulation of the homogenized limit problem

$$
\begin{aligned}
|\Gamma| \partial_{t} u_{0}-\nabla_{x} \cdot\left(S(x) \nabla_{x} u_{0}\right)+|\Gamma| u_{0} & =\int_{\Gamma} f \mathrm{~d} \sigma_{y} & & \text { in } \Omega, \\
u_{0} & =0 & & \text { on } \partial \Omega .
\end{aligned}
$$

Acknowledgements We would like to thank Bernd Schmidt for fruitful discussions related to Lemma 5.

\section{References}

[1] G. Allaire and A. Damlamian and U. Hornung, Two-scale convergence on periodic surfaces and applications in Proceedings of the international conference on mathematical modelling of flow through porous media, editors: A. P. Bourgeat and C. Carasso and S. Luckhaus and A. Mikelić, World Scientific, p. 15-25, 1995.

[2] T. Arbogast and J. Douglas and U. Hornung, Derivation of the double porosity model of single phase flow via homogenization theory, SIAM J. Math. Anal. 21, p. 823-836, 1990.

[3] D. Cioranescu and A. Damlamian and P. Donato and G. Griso and R. Zaki, The periodic unfolding method in domains with holes, SIAM J. Math. Anal. 44(2), p. 718-760, 2012.

[4] D. Cioranescu and A. Damlamian and G. Griso, Periodic unfolding and homogenization, Comptes Rendus Mathematique 335(1), p. 99-104, 2002.

[5] D. Cioranescu and A. Damlamian and G. Griso, The periodic unfolding method in homogenization, SIAM J. Math. Anal. 40(4), p. 1585-1620, 2008.

[6] D. Cioranescu and P. Donato and R. Zaki, The periodic unfolding method in perforated domains, Portugaliae Mathematica 63(4), p. 467-496, 2006.

[7] S. Dobberschütz and M. Böhm, A Periodic Unfolding operator on certain compact Riemannian manifolds, Comptes Rendus Mathmatique 350, p. 1027-1030, 2012.

[8] I. Graf, Multiscale modeling and homogenization of reaction-diffusion systems involving biological surfaces, PhD dissertation, Universität Augsburg, Logos Verlag Berlin, 2013.

[9] I. Graf and M. Peter, Homogenization of fast diffusion on surfaces with a two-step method and an application to T-cell signaling, Nonlinear Analysis: Real World Applications 17, p. 344-364, 2014.

[10] M. Höpker and M. Böhm, A note on the existence of extension operators for Sobolev spaces on periodic domains, preprint, submitted for publication.

[11] P. Li, Seminar on Differential Geometry, Princeton University Press, 1982.

[12] M. Mabrouk and S. Hassan, Homogenization of a composite medium with a thermal barrier, Mathematical Methods in the applied Sciences 27, p. $405-425,2004$.

[13] M. Neuss-Radu, Some extensions of two-scale convergence, C. R. Acad. Sci. Paris, Ser. I 322, p. 899-904, 1996.

[14] M. A. Peter and M. Böhm, Different choises of scaling in homogenization of diffusion and interfacial exchange in a porous medium, Mathematical Methods in the Applieds Sciences 31, p. 1257-1282, 2008. 